Current Perspectives on Medicinal \& Aromatic Plants

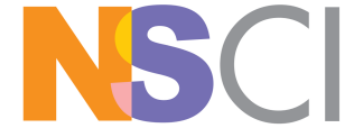

\title{
Medicinal Plants of Bulgaria
}

\author{
Alexander Tashev*, Violeta Dimitrova \\ University of Forestry, Faculty of Forestry, Department of Dendrology, 10 Kliment Ohridski Blvd., 1756 \\ Sofia, Bulgaria \\ *Correspondence: altashev@abv.bg, (Alexander Tashev)
}

Published: 29 July 2019

\begin{abstract}
Bulgaria is a small country in Southeast Europe, but its geographic location, varied relief and climate contribute to great floristic diversity. The medicinal plants are part of it. They represent $23.2 \%$ of the country's flora. The aim of the present paper is to present information about the full composition of the medicinal plants of Bulgarian flora as well as characteristics of the systematic structure and classification according to biological type, life form, floristic geolements, period of flowering, and ecological groups. The complete list of medicinal plants in Bulgaria was compiled on the basis of the Medicinal Plants Act, extensive literature analysis and own researches. The life forms were identified according to Raunkiaer and for floral elements the classification of Walter was used. The ecological forms were identified according to Flora of Republic Bulgaria. As a result of the conducted surveys it was found that there are 950 medicinal species of vascular plants in the country belonging to 468 genera and 126 families. The richest family containing medicinal plants was Rosaceae (98 specie). The most medicinal plants existed among perennial herbaceous plants (539 species). Geoelements with a European component were predominated (408 species). The most plants bloom from June to August (121 species). The ecological structure of medicinal plants shows that mesophytes (435 species); heliophytes (545 species) and thermophylous plants (607 species) were dominated. The results of the study include also a detailed information about the national and international conservation importance of the analyzed species. 65 species were included in the Red Book of Bulgaria. There were 81 species protected by the Biodiversity Law. Among the medicinal plants are 16 Balkan and 4 Bulgarian endemic spesies. The international significance of these plants was defined by the fact that 33 of species were under protection by different international conventions. The ratio between the biological types, the predominant geoelements and the life forms shows is typical for the temperate continental climate zone and the flora of our country. The total percentage of protected species $(7 \%)$ is not high, which is positive because most species are subject to use by the local population, the pharmaceutical and perfumery industry.
\end{abstract}

Key words: Medicinal plants, flora, systematic structure, geoelements, biological types, conservation significance, Bulgaria

\section{Introduction}

Bulgaria is a small country in Southeast Europe, but its geographic location, varied relief and climate contribute to great floristic diversity. The medicinal plants are part of it. They represent $23.2 \%$ of the country's flora. Regardless of the great achievements of organic synthetic chemistry in the field of pharmaceuticals, the interest in medicinal plants and medicines obtained from them does not decrease. On the contrary, he has shown a certain upswing in recent years.

In our country many authors have focused their research on medicinal plants (Stoyanov, 1972, Ivanov et al, 1973, Hardalova et al., 1994, Assenov et al., 1998, Gussev, 2005, 
Landzhev, 2005, Evstatieva et al., 2007, Vitkova and Tahsev, 2007, Nikolov, 2007, Tashev and Tsavkov, 2008, Koev et al., 2014). The aim of the present paper is to present information about the full composition of the medicinal plants of Bulgarian flora as well as characteristics of the systematic structure and classification according to biological type, life form, floristic geolements, period of flowering, and ecological groups.

\section{Material and Methods}

Study sites: Bulgaria is a small country in Southeast Europe (Fig. 1) with a territory of 110,994 square kilometres. On this territory there is a mountainous, hilly and plain relief. Bulgaria has a changeable climate, which results from being positioned at the meeting point of the Mediterranean and continental air masses combined with the barrier effect of mountains and nearness of Black Sea. The interaction of climatic, hydrological, geological and topographical conditions has produced a relatively wide variety of plant species. Bulgaria's biodiversity is one of the richest in Europe. According to the latest official data, 4102 species of higher plants from 913 genera and 155 families have been established in the flora of Bulgaria (Assyov and Petrova, 2012).

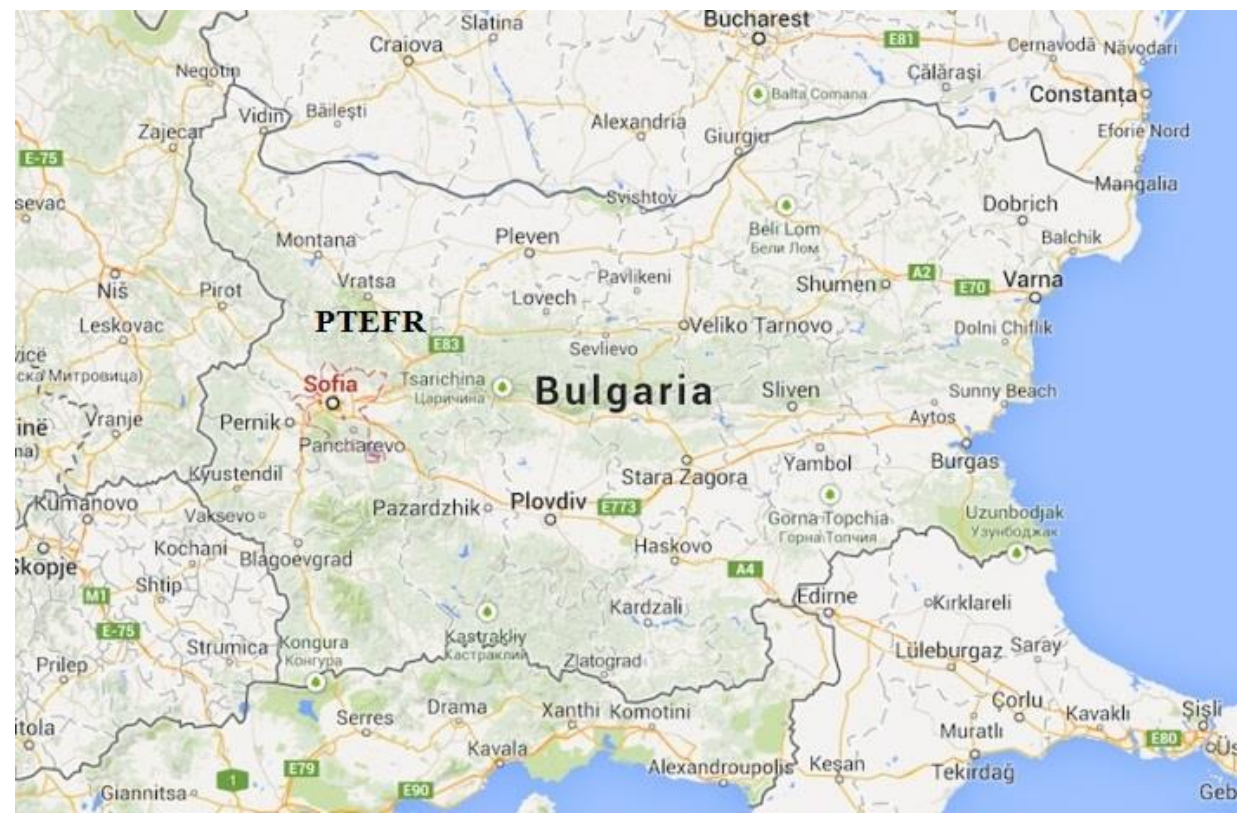

Fig. 1. Object of investigation

The complete list of medicinal plants in Bulgaria was compiled on the basis of the Medicinal Plants Act, extensive literature analysis and own researches. The life forms were identified according to Raunkiaer,1934 and for floral elements the classification of Walter [14] was used. The ecological forms were identified according to Flora of Republic Bulgaria (Stoyanov, 1966, Jordanov, 1963, Velchev, 1989, Kozhuharov, 1995, Peev, 2012). The following documents are used to determine the conservation significance of the species: Biological Diversity Law [20]; Convention on International Trade in Endangered Species (CITES); Convention on the Conservation of European Wildlife and Natural Habitats. Appendix I.; Council Directive 92/43/EEC, 21.05.1992 on the protection of natural habitats and wild fauna and flora; IUCN Red List of Threatened Plants. 


\section{Results}

In this paper, for the first time, an attempt has been made to characterize all the medicinal plants known in Bulgaria. Analysis of literature data, and own research, found that in official and traditional medicine and in homeopathy in Bulgaria, 950 species belonging to 468 genera and 126 families are used (Annex 1). This represents $23.2 \%$ of all species, $51.3 \%$ of the genera and $81.3 \%$ of all vascular plant families in the country. The systematic structure of these plants shows that 4 species from 4 genera and 2 families belong to Lycopodiophyta, 5 species from one genus and one family belong to Equisetophyta, 14 species from 10 genera and 7 families belong to Polypodiophyta. Pinophyta is represented by 12 species from 7 genera and 4 families, Magnoliophyta 915 species from 446 genera and 112 families of which 98 species from 46 genera and 17 families belong to Liliopsida, and to Magnoliopsida - 817 species from 400 genera and 95 families. The richest families containing medicinal plants are Rosaceae - 98 species, Lamiaceae - 86 species, Asteraceae - 80 species, Apiaceae - 51 species, Fabaceae - 50 species, Brassicaceae - 49 species, Ranunculaceae and Scrophulariaceae - 29 species, Alliaceae - 25 species, Orchidaceae and Polygonaceae - 19 species, etc. Families represented by only one species are 37.

The distribution of taxa by biological types shows that the largest number of medicinal plants is of herbaceous perennials - 539 species or $56.8 \%$ of all plants, followed by annuals - 130 species (13.7\%), shrubs - 91 species (9.6\%) and trees - 48 species (5.0\%). The remaining species belong to the transitional biological types. The biological spectrum of medicinal plants [13] is dominated by hemicryptophytes $(\mathrm{H})-363$ species or $38.2 \%$ of the total, followed by cryptophytes (Cr) - 176 species (18.5\%), phanerophytes (Ph) - 155 species (16.4\%), terophytes (Th) - 130 species $(13.6 \%)$ and hamefites $(\mathrm{Ch})-35$ species $(3.6 \%)$. The remaining species belong to transitional life forms - among them most are 50 species (5.2\%), these are plants that can be hemicryptophytes or terophytes (H-Th), depending on the habitat. An analysis of the distribution of medicinal plants by the geo-elements of the flora according to the Walter classification adapted for the flora of Bulgaria [11] shows that the geo-elements with the European component are predominant - 408 species, or $42.9 \%$. Among them, the most Eurasian (Eur-As) are 136 species (14.3\%), Euro-Mediterranean (Eur-Med) - 92 species (9.7\%), European (Eur) 76 species (8.1\%), Euro-Siberian (Eur-Sib) - 73 species $(7.7 \%)$, etc. On second place is a group of species with a mediterranian component - 333 species, or $35.0 \%$. Most of them are submediterranian (subMed) - 120 species $(12.7 \%$ ), Euromediterranian (Eur-Med) - 92 species (9.7\%), Mediterranean (Med) itself - 55 species (5.8\%), Ponti-Tertiary Terran (Pont-Med) - 30 species (3.2\%), EuroSubmediterans (Eur-subMed) - 18 species (1.9\%), etc. Boreal species (Boreal) are 112 (11.8\%), adventitious (Adv) - 76 (8.1\%), and cosmopolitan (Kos) - 30 (3.2\%), etc. For two species (Allium longispathum Redoute and A. webbii GC Clementi) there is no information on the florogenetic affiliation. The distribution of medicinal plants by flowering period shows that the most plants bloom from June to August - 121 species (12.7\%). 96 species bloom in June-July, 85 species - from May to July, 84 - from June to September, 81 - from June to August, 71 - from May to June, etc.

An ecological characteristic of medicinal plants has been made in relation to three main environmental factors: moisture, solar lightening and temperature. In relation to the moisture factor, mesophytes predominate, represented by 435 species $(45.8 \%)$, 
followed by xeromezophytes - 139 species, xerophytes - 135 species, hygromezophytes - 94 species, hygrophytes - 92 species and in the last hydrophytes -21 species. Heliophytes dominate in relation to the light factor - there are more than half of all species - 545 (57.4\%), the hemiscyophyte group includes 269 plants $(28.3 \%)$, and sciophytes - 136 species (14.3\%). In relation to the thermal factor, the species are divided into three groups. More than half are thermophilic plants - 607 species $(63.9 \%)$, followed by mesotherms - 247 species (26.0\%), and the remaining 96 species $(10.1 \%)$ belong to the group of microtherms. An analysis of the conservation value of medicinal plants, made on the basis of national and international reference documents, shows the following: the Red Book of the Republic of Bulgaria (Peev, 2015), includes a total of 65 species of which 2 (Hottonia palustris L., Pimpinella major (L.) Huds.) with category "regionally extinct species" (Re), 24 species with category "under critical threat of extinction" (Cr), 36 species with category "under threat of extinction" (En) and 3 species with category "vulnerable" (Vu). Under the protection of the Biodiversity Law there are 81 species. Among the medicinal plants are 16 Balkan and 4 Bulgarian endemics. The international significance of these plants is determined by the fact that 22 species are protected by the Convention on International Trade in Endangered Species of Wild Fauna and Flora, 4 species (Campanula lanata Friv., Orchis provincialis Balb., Rheum rhaponticum L., Vaccinium arctostaphylos L.) are under strict protection of the "Convention on the Conservation of European Wildlife and Natural Habitats" (Bern Convention).

Annex II of Directive 92/43/EEC on the protection of natural habitats and wild fauna and flora includes 2 species (Echium russicum JF Gmel., Himantoglossum caprinum (M. Bieb.) Spreng.), five species (Alchemilla achtarowii Pawl., A. asteroantha Rothm., A. jumrukczalica Pawl., Campanula lanata, Rheum rhaponticum) are included in the "IUCN Red List of Threatened Plants" (Walter and Gillett (eds), 1998).

\section{Discussion and Conclusion}

The ratio between the biological types, the predominant geoelements and the life forms shows that it is typical for the temperate continental climate zone and the flora of our country. The total percentage of protected species (7\%) is not high, which is positive because most species are subject to use by the local population, the pharmaceutical and perfumery industry.

\section{Annex I. Sistematic list of medicinal plants of Bulgarian flora}

\section{Lycopodiophyta}

Lycopodiaceae: Diphasiastrum alpinum (L.) Holub., Huperzia inundata (L.) Bernh ex Schrank et Mart., Lycopodium clavatum L.; Ophioglossaceae: Ophioglossum vulgatum L.

\section{Equisetophyta}

Equisetaceae: Equisetum arvense L., E. fluviatile L., E. palustris L., E. sylvaticum L., E. telmateia Ehrh.

\section{Polypodiophyta}

Adiantaceae: Adiantum capillus-veneris L.; Aspidiaceae: Dryopteris filix-mas (L.) Schott., Polystichum lonchitis (L.) Roth.; Aspleniaceae: Asplenium adiantum-nigrum L., A. ruta- 
muraria L., A. septentrionale (L.) Hoffm., A. trichomanes L., Ceterach officinarum DC., Phyllitis scolopendrium (L.) Newm.; Athyriaceae: Athyrium filix-femina (L.) Roth.; Hypolepidaceae: Pteridium aquilinum (L.) Kuhn.; Osmundaceae: Osmunda regalis L.; Polypodiaceae: Polypodium cambriacum L., P. vulgare L.

\section{Pinophyta}

Cupressaceae: Juniperus communis L., J. excelsa Bieb., J. oxycedrus L., J. sabinaL., J. sibirica Burget.; Ephedraceae: Ephedra distachya L.; Ginkgoaceae: Ginkgo biloba L. Pinaceae: Abies alba Mill., Picea abies (L.) Karst., Pinus nigra Arnold, P. peuce Griseb., P. sylvestris L.; Taxaceae: Taxus baccata $\mathrm{L}$.

\section{Magnoliphyta \\ Liliopsida}

Alismataceae: Alisma plantago-aquatica L.; Alliaceae: Allium albidum Fischer ex Bieb., A. amethystinum Tausch, $A$. ampeloprasum L., A. angulosum L., A. atroviolaceum Boiss., $A$. carinatum L., A. cepa L., A. cirrhosum Vand., A. flavum L., A. fuscum Waldst. \& Kit.

A. guttatum Steven, A. longispathum Redoute, A. melanantherum Pančič, A. paczoskianum Tuzson, A. porrum L., A. proliferum (Moench) Schrader, A. rotundum L., A. sativum L., $A$. schaerocephalon L., A. schoenoprasum L., A. scordoprasum L., A. ursinum L., A. victorialis L., A. webbii G. C. Clementi, Nectaroscordum siculum (Ucria) Lindley; Amaryllidaceae: Galanthus elwesii Hook., G. nivalis L., Leucojum aestivum L.; Araceae: Acorus calamus L., Arum italicum Mill., A. maculatum L., Dracunculus vulgaris Schott; Asparagaceae: Asparagus officinalis L., A. tenuifolius Lam., Ruscus aculeatus L.; Butomaceae: Butomus umbellatus L.; Cyperaceae: Eriophorum angustifolium Honck., E. latifolium Hoppe., E. vaginatum L.; Dioscoreaceae: Tamus communis L.; Hydrocharitaceae: Hydrocharis morsus-ranae L.; Iridaceae: Crocus chrysanthus Herb., C. pallasii Bieb., Gladiolus communis L., G. imbricatus L., Iris graminea L., I. pseudacorus L., I. pumila L.; Juncaceae: Juncus inflexus L.; Lemnaceae: Lemna minor L., Spirodela polyrhiza (L.) Schleid.; Liliaceae: Asphodeline lutea (L.) Reichenb., Asphodelus albus Mill., Colchicum autumnale L., C. diampolis Delip. \& Česchm., Convallaria majalis L., Lilium martagon L., Paris quadrifolia L., Polygonatum latifolium (Jacq.) Desf., P. multiflorum (L.) All., P. odoratum (Mill.) Druce, Scilla bifolia L., Veratrum lobelianum Bernth., V. nigrum L.; Najadaceae: Najas marina L.; Orchidaceae: Anacamptis pyramidalis (L.) L.C. Rich., Gymnadenia conopsea (L.) R. Br., Himantoglossum hircinum (L.) Spreng., Orchis coriophora L., $O$. laxiflora Lam., O. mascula L., O. militaris L., O. morio L., O. pallens L., O. papilionacea L., $O$. provincialis Balb., O. punctulatum Stev., O. purpurea Huds., O. simia L., O. spitzelii Saut. ex Koch., O. tridentata Scop., O. ustulata L., Platanthera bifolia (L.) L.C. Rich., P. chlorantha (Cust.) Rehb.; Poaceae: Anthoxanthum odoratum L., Briza media L., Cynodon dactylon (L.) Pers., Elymus repens (L.) Gould., Hierochloe australis (Schrader) Roemer \& Schultes, Hordeum vulgare L., Lolium temulentum L. Sclerochloa dura (L.) Beauv., Zea mays L.; Sarganiaceae: Sparganium erectum L.; Smilacaceae: Smilax excelsa L.; Typhaceae: Typha angustifolia L., T. latifolia L.

\section{Magnolipsida}

Acanthaceae: Acanthus balcanicus Heyw. et Richards, A. spinosus L.; Aceraceae: Acer campestre L., A. negundo L., A. platanoides L., A. pseudoplatanus L., A. tataricum L.; Adoxaceae: Adoxa moschatellina L.; Amaranthaceae: Amaranthus spinosus L.; Anacardiaceae: Cotinus coggygria Scop., Pistacia vera L., Rhus coriaria L.; Apiaceae: Aethusa cynapium L., Anethum graveolens L., Angelica archangelica L., A. pancicii Vand., A. 
sylvestris L., Anthriscus cerefolium (L.) Hoffm., Apium graveolens L., Astrantia major L., Bifora tadians Bieb., Bupleurum longifolium L., B. rotundifolium L., Carum carvi L., C. graecum Boiss.et Heldr., Chaerophyllum bulbosum L., C. tenulentum L., Cicuta virosa L., Conium maculatum L., Coriandrum sativum L., Cuminum cyminum L., Daucus sativus Hoffm., Eryngium campestre L., E. maritimum L., Ferulago sylvatica (Bess.) Rchb., Foeniculum vulgaris Mill., Heracleum sibiricum L., H. verticillatum Panc., Laser trilobum (L.) Borkh., Laserpitium latifolium L., L. prutenicum L., L. siler L. Levisticum officinale Koch, Meum athamanticum Jacq., Oenanthe aquatica L., Opopanax chironium (L.) Koch., $O$. hispidus (Friv.) Grsb. Petroselinum crispum (Mill.) A. W. Hill, Peucedanum arenarium Waldst. \& Kit., P. cervaria (L.) Lapeyer., P. officinalis L., P. ruthenicum Bieb., Pimpinella anisum L., P. major (L.) Huds., P. saxifraga L., Prangos ferulacea (L.) Lindl., Sanicula europaea L., Scandix pecten-veneris L., Seseli rigidum W. et K., S. tortuosum L., Tordylium maximum L., Torilis arvensis (Huds.) Link; Apocynaceae: Trachomitum venetum (L.) Woodson, Vinca herbacea W. et K., V. major L., V. minor L.; Aquifoliaceae: Ilex aquifolium L., I. colchica Pojark.; Araliaceae: Hedera helix L.; Aristolochiaceae: Aristolochia clematitis L., A. rotunda L., Asarum europaeum L.; Asclepiadaceae: Cionura erecta (L.) Griseb., Periploca graeca L., Vincetoxicum fuscatum (Hornem.) Reichenb. f., V. hirundinaria Medic., V. speciosum Boiss. \& Spruner; Asteraceae: Achillea clypeolata Sm., A. grandifolia Friv., A. millefolium L., A. nobilis L., Antennaria dioica (L.) Gaertn., Anthemis cotula L., A. tinctoria L., Arctium lappa L., A. minus Bernh., $A$. nemorosum L., $A$. tomentosum Mill., Artemisia absinthium L., A. alba Turra, A. annua L., A. campestre L., $A$. dracunculus L., A. lerchiana Weber, A. santonicum L., A. vulgaris L., Bellis perennis L., Bidens tripartita L., Calendula officinalis L., Carduus acanthoides L., C. armatus Boiss. \& Heldr., Carlina acanthifolia All., C. vulgaris L., Carthamus lanatus L., Centaurea calcitrapa L., C. cyanus L., C. pannonica (Heuff.) Simk., C. rocheliana (Heuff.) Dost., C. solstitialis L., Chamomilla recutita (L.) Rausch., C. suaveolens (Pursh.) Rydb., Cichorium endivia L., C. intybus L., Cnicus benedictus L., Doronicum columnae Ten., Echinops sphaerocephalus L., Filago lutescens Jord., F. vulgaris Lam., Galinsoga parviflora Cav., Gnaphalium uliginosum L., Helichrisum arenariun (L.) Moench., Hieracium pilosella L., Hypochaeris maculata L., $H$. radicata L., Inula aschersoniana Janka, I. ensifolia L., I. germanica L., I. helenium L., Lactuca serriola L., Leucanthemum vulgare Lam., Ligularia glauca (L.) O. Hoffm., Matricaria trichophylla (Boiss.) Boiss., Onopordium acanthium L., O. tauricum Willd., Petasites albus (L.) Gaertn., P. hybridus (L.) Gaertn., Pulicaria dysentherica (L.) Bernh., P. vulgaris Gaerth., Scorzonera hispanica L., Senecio jacobaea L., S. nemorensis L., S. othonnae Bieb., S. paludosus L., S. viscosus L., S. vulgaris L., Silybum marianum (L.) Gaerth., Solidago virgaurea L., Tagetes erecta L., T. minima L., Tanacetum vulgare L., Taraxacum officinale Web., Telekia speciosa (Schreb.) Baumg., Tragopogon pratensis L., Tussilago farfara L., Xanthium spinosum L., X. strumarium L., Xeranthemum annuum L.; Balsaminaceae: Impatiens balsamina L., I. parviflora DC., Berberis thunbergii DC., B. vulgaris L.; Betulaceae: Alnus glutinosa (L.) Gaertn., A. incana (L.) Moench, Betula pendula Roth.; Boraginaceae: Alkanna tinctoria (L.) Taush, Anchusa azurea Mill., A. officinalis L., Buglossoides arvensis (L.) Lohnst., B. purpurocaerulea (L.) Lohnst., Cerinthe glabra Mill., C. minor L., Cynoglossum officinale L., Echium italicum L., E. russicum J. Gmel., E. vulgare L., Heliotropium europaeum L., Lithospermum officinale L., Pulmonaria mollis Wulf. et Horn., P. officinalis L., Symphytum officinale L.; Brassicaceae: Alliaria petiolata (Bieb.) Cavara et Grande., Alyssum alyssoides L., Armoratia macrocarpa (Waldst. \& Kit.) Kit. ex Baumg., A. rusticana (Lam.) P. Gaerthner, B. Meyer \& Scherb., Barbarea vulgaris R.Br., Brassica juncea (L.) Czern., B. nigra (L.) Koch., Bunias orientalis L., Capsella bursa-pastoris (L.) Medic., Cardamine amara L., C. bulbifera (L.) Crantz., C. pratensis L., Coronopus 
procumbens Gilib., Descurainia sophia (L.) Webb. ex Prantl., Diplotaxis tenuifolia (L.) DC., Euclidium syriacum (L.) R.Br., Hesperis matronalis L., Lepidium campestre (L.) R. Br., L. graminifolium L., L. latifolium L., L. perfoliatum L., L. ruderale L., L. sativum L., Lunaria rediviva L., Nasturtium officinalis $\mathrm{R}$. Br., Raphanus raphanistrum L., Rorippa austriaca (Crantz.) Bess., R. sylvestris (L.) Bess., Sinapis alba L., Sisymbrium loeselii L., S. officinale (L.) Scop., Thlaspi alliaceum L., T. arvense L.; Buddlejaceae: Buddleja davidii Franchet; Buxaceae: Buxus sempervirens L.; Campanulaceae: Campanula lanata Friv., C. persicifolia L., Jasione heldreichii Boiss. \& Orph., J. montana L.; Cannabaceae: Cannabis sativa L., Humulus lupulus L.; Caprifoliaceae: Lonicera caprifolium L., L. xilosteum L., Sambucus deborensis (Košamin) Košamin, S. ebulus L., S. nigra L., S. racemosa L., Viburnum opulus L.; Caryophyllaceae: Agrostemma githago L., Dianthus giganteiformis Borbas, D. pontederae A.Kern., Gypsophila paniculata L., Herniaria glabra L., H. hirsuta L., H. incana Lam., Lychnis coronaria (L.) Desr., L. flos-cuculi L., Minuartia setacea (Thuill) Hay., Saponaria officinalis L., Scleranthus annuus L., S. perennis L., Silene otites (L.) Wibel., Spergularia rubra (L.) J. et C. Prest., Stellaria graminea L., S. media (L.) Vill., Viscaria vulgaris Röhling; Celastraceae: Euonymus europaeus L., E. verrucosus Scop.; Chenopodiaceae: Atriplex rosea L., Camphorosma monspeliaca L., Chenopodium album L., C. ambrosioides L., C. bonus-henricus L., C. botrys L., C. foliosum (Moench) Ascherson, C. hybridum L., C. polyspermum L., C. rubrum L., C. vulvaria L., Salicornia europaea L., Salsola soda L.; Cistaceae: Cistus incanus L., Helianthemum nummularium (L.) Mill.; Convolvulaceae: Calystegia sepium (L.) Rr., Convolvulus arvensis L.; Cornaceae: Cornus mas L., C. sanguinea L.; Corylaceae: Carpinus betulus L., C. orientalis Mill., Corylus avellana L., C. colurna L.; Crassulaceae: Jovibarba heuffelii (Schott) A. \& D. Löve, J. velenovskyi (Česchm.) J. Holub, Rhodiola rosea L., Sedum acre L., S. album L., S. maximum (L.) Suter., S. spectabile Boreau, Sempervivum erythraeum Velen., S. leucanthum Pančič, $S$. marmoreum Grab., S. tectorum L., S. zeleborii Schott, Umbilicus erectus DC.; Cucurbitaceae: Bryonia alba L., B. cretica ssp. dioica (Jacq.) Tutin, Citrullus colocynthis (L.) Schrader, C. lanatus (Thumb.) Matsum. \& Nakai, Cucurbita maxima Duchesne, Ecbalium elaterium (L.) A. Rich.; Cuscutaceae: Cuscuta epilinum Weihe, C. europaea L.; Dipsacaceae: Dipsacus fullonum L., Knautia arvensis (L.) Coult., Scabiosa ochroleuca L., Succisa pratensis Moench.; Droseraceae: Drosera rotundifolia L.; Elaeagnaceae: Elaeagnus angustifolia L., Hippophae rhamnoides L.; Ericaceae: Arctostaphylos uva- ursi (L.) Spreng., Calluna vulgaris (L.) Hull., Rhododendron ponticum L., Vaccinium arctostaphylos L., V. myrtillus L., V. uliginosum L., V. vitis-idaea L.; Euphorbiaceae: Euphorbia amygdaloides L., E. cyparissias Host., E. myrsinites L., E. peplis L., E. peplus L., Mercurialis annua L., M. perennis L., Ricinus communis L.; Fabaceae: Amorpha fruticosa L., Anthyllis vulneraria L., Astragalus dasyanthus Pallas, A. glycyphylloides DC., A. glycyphyllos L., Bituminaria bituminosa (L.) Stirt., Cercis siliquastrum L.; Fabaceae: Chamaecytisus albus (Jacq.)Rothm., C. hirsutus (L.) Link., C. lejocarpus (A. Kern.) Rothm., C. ratisbonensis (Schaeff.) Rothm., Chamaespartium sagittale (L.) P. Gibbs., Colutea arborescens L., Coronilla scorpioides (L.) Koch., C. varia L., Galega officinalis L., Genista ovata W.et K., G. tinctoria L., Gleditsia triacanthos L., Glycyrrhiza glabra L., Hippocrepis comosa L., Lathyrus niger (L.) Bernh., L. pratensis L., L. sativus L., L. sylvesris L., L. tuberosus L., L. vernus (L.) Bernh., Lotus corniculatus L., Medicago disciformis DC., Melilotus alba Medic., M. indica (L.) All., M. officinalis (L.) Pal., Ononis pusilla ssp. orientalis (Širj.) Kožuharov, O. spinosa L., Robinia pseudoacacia L., Securigera securidaca (L.) Roth., Spartium junceum L., Tetragonolobus maritimus (L.) Roth., Trifolium alpestre L., T. arvense L., T. pannonicum Jacq., T. pratense L., T. repens L., Trigonella coerulea (L.) Ser., T. foenum-graecum L., T. procumbens (Bess.) Rchb., Vicia cracca L., V. grandiflora Scop., V. 
pisiformis L., V. sativa L.; Fagaceae: Castanea sativa Mill., Fagus orientalis Lipsky, F. sylvatica L., Quercus coccifera L., Q. frainetto Ten., Q. robur L.; Fumariaceae: Corydalis bulbosa (L.) DC., C. solida (L.)Swartz., Fumaria officinalis L., F. vaillantii Loisel.; Gentianaceae: Centaurium erythraea Rafn., C. pulchellum (Swartz) Druce, Gentiana asclepiadea L., G. cruciata L., G. lutea L., G. pneumonanthe L., G. punctata L., G. verna L., Gentianella bulgarica (Vel.) Holub.; Geraniaceae: Erodium cicutarium (L.) L'Her., Geranium macrorrhizum L., G. pyrenaicum Burm. f., G. robertianum L., G. sanguineum L., G. sylvaticum L.; Gesneriaceae: Haberlea rhodopensis Friv.; Globulariaceae: Globularia ahyllanthes Crantz, G. cordifolia L.; Grossulariaceae: Ribes nigrum L., $R$. uva-crispa L.; Haloragaceae: Myriophyllum spicatum L.; Hippocastanaceae: Aesculus hippocastanum L.; Hypericaceae: Hypericum androsaemum L., H. cerastoides (Spach) N. Robson, $H$. elegans Stephan ex Willd., H. hirsutum L., H. maculatum Crantz., $H$. olympicum L., $H$. perforatum L.; Juglandaceae: Juglans regia L.; Lamiaceae: Acinos alpinus (L.) Moench, A.arvensis (Lam.) Dandy, A. suaveolens (S.et S.) G.Don., Ajuga chamaepitis (L.) Schreb., A. laxmannii (L.) Benth., A. reptans L., Ballota nigra L., Betonica officinalis L., Calamintha grandiflora (L.) Moench, C. nepeta (L.) Savi., Clinopodium vulgare L., Galeopsis ladanum L., G. speciosa Mill., G. tetrachit L., Glechoma hederaceae L., G. hirsuta Waldst. \& Kit., Hyssopus officinalis L., Lamium album L., L. maculatum L., L. purpureum L., Lavandula angustifolia Mill., Leonurus cardiaca L., Lycopus europaeus L., Marrubium parviflorum Friv. et Mey., M. peregrinum L., M. vulgare L., Melissa officinalis L., Melittis melissophylum L., Mentha aquatica L., M. arvensis L., M. longifolia (L.) Huds., M. piperita L., M. pulegium L., M. spicata L., M. suaveolens Ehrh., M. verticillata L., Nepeta cataria L., Ocinum basilicum L., Origanum vulgare L., Phlomis tuberosa L., Prunella grandiflora (L.) Scholler, P. vulgaris L., Rosmarinus officinalis L., Salvia aethiopis L.. S. glutinosa L., S. nemorosa L., S. officinalis L., S. pratensis L., S. sclarea L., S. tomentosa Mill., S. verticillata L., Satureja cuneiflora Ten., S. montana L., Scutellaria altissima L., S. galericulata L., S. hastifolia L., Sideritis montana L., S. scardica Griseb., S. syriaca L., Stachys annua L., S. germanica L., S. recta L., S. sylvatica L., Teucrium chamaedrys L., T. montanum L., T. polium L., T. scordium L., Thymus albanus H. Braun, T. atticus Čelak., T. bracteosus Vis. ex Bentham, T. callieri Borb., T. comptus Friv., T. glabrescens Willd., T. jankae Čelak., T. leucotrichus Halacsy, T. longicaulis C. Presl., T. longidentatus (Deg. et Urum.) Ronn., T. moesiacus Velen., T. pannonicus All., T. perinicus (Velen.) Jalas, T. pulegioides L., T. sibthorpii Benth., T. stojanovii Degen, T. striatus Vahl., T. thracicus Velen., T. vandasii Velen., T. zygioides Griseb.; Linaceae: Linum catharticum L., L. usitatissimum L.; Loranthaceae: Loranthus europaeus Jacq., Viscum album L.; Lythraceae: Lythrum salicaria L., L. virgatum L.; Malvaceae: Abutilon theophrastii Medic., Alcea pallida (Waldst.) Waldst. \& Kit., A. rosea L., Althaea cannabina L., A. officinalis L., Gossypium hirsutum L., Hibiscus syriacus L., H. trionum L., Lavatera thuringiaca L., Malva alcea L., M. moschata L., M. neglecta Wallr., M. pusilla Sin., M. sylvestris L.; Menyanthaceae: Menyanthes trifoliata L.; Monotropaceae: Monotropa hypopitys L.; Moraceae: Ficus carica L., Morus alba L., M. nigra L.; Nymphaeaceae: Nuphar lutea (L.) S. et S., Nymphaea alba L.; Oleaceae: Fraxinus excelsior L., F. ornus L., F. oxycarpa Willd., F. pallisiae Wilm., Jasminum officinale L., Ligustrum vulgare L., Phillyrea latifolia L., Syringa vulgaris L.; Onagraceae: Epilobium lanceolatum Sebastiani \& Mauri, E. parviflorum Scheber, Oenothera biennis L.; Orobanchaceae: Orobanche minor Sm., $O$. rapum-genistae Thuill.; Oxalidaceae: Oxalis acetosella L.; Paeoniaceae: Paeonia peregrina Mill.; Papaveraceae: Chelidonium majus L., Glaucium corniculatum (L.) J. H. Rudolph, G. flavum Crantz., G. leiocarpum Boiss., Papaver rhoeas L., P. somniferum L.; Parnassiaceae: Parnassia palustris L.; Phytolaccaceae: Phytolacca americana L.; Plantaginaceae: Plantago afra L., P. coronopus L., P. lanceolata L., P. major L., P. media L., 
P. scabra Moench., P. subulata L.; Platanaceae: Platanus orientalis L.; Plumbaginaceae: Limonium vulgare Mill., Plumbago europaea L.; Polygalaceae: Polygala amarella Crantz., P. major Jacq., P. monspeliaca L., P. vulgaris L.; Polygonaceae: Bistorta major S. Gray., Fagopyrum esculentum Moench, Oxyria digyna (L.) Hill., Persicaria hydropiper (L.) Spach., P. lapathifolia (L.) S. Gray, P. maculata (Raf.) S. Gray, Polygonum arenastrum Boreau, $P$. aviculare L., Rheum rhaponticum L., Rumex acetosa L., $R$. acetosella L., $R$. alpinus L., $R$. aquaticus L., $R$. crispus L., $R$. hydrolapathum Huds., $R$. obtusifolius L., $R$. patientia L., $R$. pulcher L., R. scutatus L.; Portulacaceae: Portulaca oleracea L.; Primulaceae: Anagallis arvensis L., Cyclamen coum Mill., C. hederifolim Ait., Hottonia palustris L., Lysimachia nummularia L., Primula acaulis (L.) L., P. elatior (L.) Hill., P. veris L., Samolus valerandi L.; Punicaceae: Punica granatum L.; Pyrolaceae: Moneses uniflora (L.) Gray., Orthilia secunda (L.) House, Pyrola chlorantha Swartz., P. rotundifolia L.; Ranunculaceae: Aconitum licoctonum ssp. neapolitanum (Ten.) Nym., $A$. firmum Reichenb., $A$. variegatum L., Actaea spicata L., Adonis aestivalis L., A. vernalis L., Anemone nemorosa L., $A$. ranunculoides L., Aquilegia nigricans Baumg., Caltha palustris L., Clematis recta L., C. vitalba L., Consolida hispanica (Costa) Greut. et Burdet., C. regalis S.F.Gray., Ficaria verna Huds., Helleborus odorus W. et K., Hepatica nobilis Mill., Isopyrum thalictroides L., Nigella arvensis L., $N$. damascena L., $N$. sativa L., Ranunculus flammula L., $R$. lingua L., $R$. polyanthemos L., $R$. repens L., $R$. scleratus L., Thalictrum aquilegifolium L., $T$. flavum L., $T$. minus L.; Resedaceae: Reseda inodora Rchb., R. lutea L.Rhamnaceae: Frangula alnus Mill., Paliurus spina-christi Mill., Rhamnus alpinus L., $R$. catharticus L., $R$. saxatilis L., Zizyphus jujuba Mill.; Rosaceae: Agrimonia eupatoria L., A. odorata All., Alchemilla achtarowii Pawl., A. acutiloba Opiz., A. anisiaca Wettst., A. asteroantha Rothm., A. bulgarica Rothm., A. catachnoa Rothm., A. cinerea Buser., A. connivens Buser., A. crinita Buser., A. erythropoda Juz., A. fissa Gunt. et Schum., A. flabellata Buser., A. glabra Neygenf., A. glaucescens Wallr., A. gorcensis Pawl., A. gracilis Opiz., A. gracillima Rothm., A. grossidens Buser., A. incisa Buser., A. indivisa (Buser.) Rothm., $A$. jumrukczalica Pawl., A. mollis (Buser.) Rothm., A. monticola Opiz., A. obtusa Buser., A. pawlowskii Assen., A. plicata Gand., A. pyrenaica Dufour., A. reniformis Buser., A. subcrenata Buser., A. viridiflora Rothm., A. xathochlora Rothm., Amygdalus communis L., A. nana L., Aronia prunifolia Rehder, Crataegus monogyna Jacq., C. orientalis Pallas \& Bieb., C. pentagyna W.et K.ex Willd., Dasiphora fruticosa (L.) Rydb., Dryas octopetala L., Filipendula ulmaria Maxim., F. vulgaris Moench., Fragaria vesca L., Geum coccineum S. et S., G. montanum L., G. reptans L., G. rivale L., G. urbanum L., Laurocerasus officinalis Roem., Malus sylvestris Mill., Potentilla alba L., P. apennina ssp. stojanovii Urum. \& Jav., P. argentea L., P. erecta (L.) Rausch., P. inclinata Vill., P. micrantha Ramond ex DC., P. neglecta Baumg., P. palustris (L.) Scop., P. pindicola (Nyman) Hausskn., P. reptans L., P. rupestris L., P. supina L., Prunus cerasifera Ehrh., P. cerasus L., P. domestica L., P. fruticosa Pall., P. mahaleb L., P. padus L., $P$. spinosa L., Rosa caesia Sm., R. canina L., R. caryophyllacea Bess., R. corymbifera Borkh., $R$. dumalis Bechst., R. gallica L., R. micrantha Borrer ex Sm., R. nitidula Bess., $R$. oxyodon Boiss., $R$. pendulina L., $R$. tomentosa Sm., Rubus caesius L., $R$. canescens DC., $R$. discolor Weihe \& Nees, $R$. hirtus Waldst. \& Kit., $R$. idaeus L., $R$. lloydianus Genev., $R$. macrophyllus Wehie \& Nees, $R$. sanguineus Friv., $R$. saxatilis L., $R$. thyrsanthus Focke, Sanguisorba minor Scop., S. officinalis L., Sorbus aucuparia L., S. domestica L., S. torminalis (L.) Crantz., Spiraea chamaedrifolia L., S. salicifolia L.; Rubiaceae: Cruciata laevipes Opiz., Galium aparine L., G. lucidum All., G. odoratum (L.) Scop., G. verum L., Rubia tinctorum L.; Rutaceae: Dictamnus albus L., Ruta graveolens L.; Salicaceae: Populus alba L., P. nigra L., P. tremula L., Salix alba L., S. caprea L., S. fragilis L., S. pentandra L., S. purpurea L., S. reticulata L., S. triandra L.; Saxifragaceae: Chrisosplenium alternifolium L., Saxifraga 
bulbifera L., S. rotundifolia L.; Scrophulariaceae: Digitalis ferruginea L., D. grandiflora Mill., D. lanata Ehrh., D. purpurea L., Euphrasia rostkoviana Hayne, Gratiola officinalis L., Kickxia elatine (L.) Dum., Kichxia spuria (L.) Dum., Lathraea squamaria L., Linaria vulgaris Mill., Pedicularis occulta Janka, P. palustris L., Pseudolysimachion orchideum (Crantz) Wraber, Rhinanthus minor L., Scrophularia canina L., S. nodosa L., Verbascum densiflorum Bertol., V. nigrum L., V. nobile Vel., V. phlomoides L., V. phoeniceum L., $V$. pseudonobile Stoj. et Stef., Veronica anagallis-aquatica L., V. arvensis L., V. austriaca L., $V$. beccabunga L., V. chamaedrys L., V. officinalis L., V. prostrata L.; Simaroubaceae: Ailanthus altissima (Mill.) Swingle.; Solanaceae: Atropa belladonna L., Capsicum esculentum Mill., Datura stramonium L., Hyoscyamus albus L., H. niger L., Nicandra physaloides (L.) Gaertn., Physalis alkekengi L., Solanum dulcamara L., S. luteum Mill., S. melongena L., S. nigrum L.; Staphyleaceae: Staphylea pinnata L.; Tamaricaceae: Myricaria germanica (L.) Desv., Tamarix ramosissima Ledeb., T. tetrandra Pall. et Bieb.; Thymeleaeceae: Daphne mezereum L., D. pontica L.; Tiliaceae: Tilia cordata Mill., T. platyphyllos Scop., T. rubra DC., T. tomentosa Moench.; Ulmaceae: Celtis australis L., Ulmus glabra Huds., U. minor Mill.; Urticaceae: Parietaria erecta Mert. \& Koch, P. lusitanica L., Urtica dioica L., U. urens L.; Valerianaceae: Centranthus ruber (L.) DC., Valeriana officinalis L., Valerianella coronata (L.) DC.; Verbenaceae: Verbena officinalis L., Vitex negundo L.; Violaceae: Viola hirta L., V. odorata L., V. tricolor L.; Vitaceae: Vitis sylvestris C. C. Gmelin, V. vinifera L.; Zygophyllaceae: Peganum harmala L., Tribulus terrestris L., Zygophyllum fabago L.

\section{References}

Assenov I., Gusev Ch., Kitanov G., Nikolov St. Petkov T. 1998. Gathering of Medicinal Plants. Biler, Sofia, 367 p. (in Bulgarian).

Assyov B., Petrova A. 2012. Conspectus of the Bulgarian vascular flora. Horology and floristic elements. Bulgarians Biodiversity Foundation, 492 p. (in Bulgarian).

Biological Diversity Law, SG no. 77 / 09.08.2002, amend. 98/2018, pp. 2-44.

Convention on International Trade in Endangered Species (CITES). http://www.ukcites.gov.uk/ default.asp.

Convention on the Conservation of European Wildlife and Natural Habitats. Appendix I. 1979. http://www.lkp.org.pl/pravo_html/konv_bernenska_zl.html.

Council Directive 92/43/EEC, 21.05.1992 on the protection of natural habitats and wild fauna and flora. http://docs.pravo.ru/document/view/28050421/

Evstatieva L., Hardalova, R. Stoyanova, K. 2007. Medicinal plants in Bulgaria: diversity, legislation, conservation and trade. Phytol. Balcan., 13(3): 415-427.

Gussev Ch. 2005. Characteristic of wild medicinal plants resources in Bulgaria and their sustainable management. In: Petrova, A. (ed.), Current state of Bulgarian biodiversity - problems and perspectives. Bulgarian Bioplatform, Sofia, pp. 495-508 (in Bulgarian).

Hardalova R., Evstatieva L., Gussev Ch. 1994. Characteristic of wild medicinal plants resources in Bulgaria and recommendations for their long term development. In: Sakalian, M. (ed.), National Biological Diversity Conservation Strategy. Main reports. 2:41-72. The Biodiversity Support Program. Bilvest 2000, Sofia (in Bulgarian)

Ivanov I., Landjev I., Neshev G. 1973. Medicinal Plants of Bulgaria and Their Use. Zemizdat, Sofia, 405 p. (in Bulgarian).

Jordanov D. (edt.) 1963-1979. Flora of NR Bulgaria. vol. I-VII. BAS. Sofia. (in Bulgarian)

Koev K., Georgiev S., Tashev A. 2014. Eco-biological characteristics of medicinal plants in the reserve Gorna Topchiya (River Tundzha hilly valley). Bulg. J. Agric. Sci. 20 (3): 601-606

Kozhuharov S. (ed.) 1995. Flora of Bulgaria. vol. X. BAS. Sofia., 428 p. (in Bulgarian)

Landzhev I. 2005. Encyclopaedia of the Medicinal Plants in Bulgaria. Trud Publishing House, Sofia 551p. (in Bulgarian).

Medicinal Plants Act. State Gazette (SG), issue 29/7.04.2000, amended SG No. 96/2017. (in Bulgarian).

Nikolov S. 2007. Specialized encyclopedia of medicinal plants in Bulgaria. Trud. Sofia. 566 p. (in Bulgarian) 
Peev D. (ed.) 2012. Flora Republicae Bulgaricae. vol. 11. Serdica: Aedibus Acad. Sci. Bulgaricae. 527 p. (In Bulgarian)

Peev D. (ed.) 2015. Red Book of the Republic of Bulgaria, volume 1, Plants and Mushrooms.

Raunkiaer, S. 1934. The Life Form of Plants and Statistical Plant Geography. Clarendon Press, Oxford. 632 p.

Stoyanov N., Stefanov B., Kitanov B. 1966-1967. Flora of Bulgaria. vol. I, II. Art and Sciences. Sofia, 1367 p. (in Bulgarian)

Stoyanov, N. 1972. Our Medicinal Plants. Part I, Nauka \& Izkustvo, Sofia. 336 p. (in Bulgarian).

Tashev A., Tsavkov E. 2008. Medicinal plants of the Bulgarian dendroflora. - Phytologia Balcanica, 14(2): 269-278.

Velchev V. (ed.) 1982-1989. Flora of NR Bulgaria. vol. VIII, IX. BAS. Sofia. 519 p. (in Bulgarian)

Vitkova A., Tahsev A. 2007. Study of the medicinal plants resources in Bulgaria: past, present and future. In: Materiales of the Third internationalconference. Khabarovsk, pp. 364-380.

Walter K. S., Gillett H. J. (eds) 1998. IUCN Red List of Threatened Plants. Compiled by the World Conservation Monitoring Centre. IUCN - The World Conservation Union. Gland, Switzerland and Cambridge, UK. LXIV + 862 p. 